\title{
Yoga Practice for the Management of Type II Diabetes Mellitus in Adults: A systematic review
}

\author{
Badr Aljasir', Maggie Bryson ${ }^{1}$ and Bandar Al-shehri ${ }^{2}$ \\ ${ }^{1}$ University of Ottawa, Ottawa and ${ }^{2}$ University of Toronto, Toronto, Ontario, Canada
}

\begin{abstract}
The effect of practicing yoga for the management of type II Diabetes was assessed in this systematic review through searching related electronic databases and the grey literature to the end of May 2007 using Ovid. All randomized controlled clinical trials (RCTs) comparing yoga practice with other type of intervention or with regular practice or both, were included regardless of language or type of publication. Each study was assessed for quality by two independent reviewers. Mean difference was used for summarizing the effect of each study outcomes with $95 \%$ confidence intervals. Pooling of the studies did not take place due to the wide clinical variation between the studies. Publication bias was assessed by statistical methods. Five trials with 363 participants met the inclusion criteria with medium to high risk of bias and different intervention characteristics. The studies' results show improvement in outcomes among patients with diabetes type II. These improvements were mainly among short term or immediate diabetes outcomes and not all were statistically significant. The results were inconclusive and not significant for the long-term outcomes. No adverse effects were reported in any of the included studies. Short-term benefits for patients with diabetes may be achieved from practicing yoga. Further research is needed in this area. Factors like quality of the trials and other methodological issues should be improved by large randomized control trials with allocation concealment to assess the effectiveness of yoga on diabetes type II. A definitive recommendation for physicians to encourage their patients to practice yoga cannot be reached at present.
\end{abstract}

Keywords: adult - systematic review - type II diabetes - yoga

\section{Background}

Interest in and use of complementary and alternative medicine has recently expanded in many countries around the world. Population-based studies in countries in the developed world, such as Australia, Scotland, UK, Taiwan, Singapore and the United States of America (USA), report that one-half to two-thirds of adults use complementary therapies (1-5).

Conventional medicine for individuals with diabetes has been geared toward regulating blood glucose with a combination of dietary modification, insulin and/or oral

For reprints and all correspondence: Badr Aljasir, Suite 708, 700 Sussex drive, K1N1K4, Ottawa, Canada. Tel: 0016138626262;

Fax:0016136805762; E-mail: draljasir@yahoo.com agents, maintaining ideal body weight, exercising regularly and self-monitoring blood sugar. Good glucose control can, however, be difficult for many people with diabetes, because these conventional treatment plans require changes to behavior and lifestyle (6). Due to the chronic course of the disease, the debilitation of complications and threat of death, as well as the complexities of treatment plans, people with diabetes often work proactively to manage their condition, optimize their health and alleviate complications through the use of complementary therapies (6-9).

Because of the potential threat to quality of life and the chronic nature of diabetes many people turn to complementary therapies seeking help to control the disease. That they do so is not unexpected since many people 
with chronic diseases utilize complementary therapies (10-12).

Yoga is an old, traditional, Indian psychological, physical and spiritual exercise regimen that has been studied for several decades for its role in the management of several chronic diseases including hypertension, asthma, obesity, neuromuscular diseases and psychiatric illnesses (13-19).

Additionally yoga has been studied for controlling both the symptoms and the complications associated with diabetes mellitus type II (20-33). The results from these studies suggested a statistically significant role for yoga in controlling diabetes. Furthermore, yoga practice showed a significant improvement for those diabetic patients with pre-existing complications (34). These findings suggest that diabetics may benefit from yoga's ability to improve their quality of life.

There are several hypotheses for the biological mechanisms that link the benefits of yoga to diabetes management (24). One hypothesis points to the role of stress and relaxation (35-37), while others suggest that the non-invasive nature of yoga provides excellent support and few side effects to the patients already taking medication for the disease. These hypotheses, however, have a limited generalizability as the studies they reference have small sample sizes, different types of outcome and methodological issues. These limitations should be taken into consideration before evidence based conclusions can be drawn (16).

The American Diabetes Association Position Statement (38) recommends that the use of adjuvant therapies be based on evidence from clinical research and presently there is no comprehensive, systematic review in the existing literature that addresses the effectiveness of yoga practice in diabetes mellitus management. A review such as this is urgently needed as type II diabetes is fast becoming one of the leading disabling diseases worldwide (39). In addition this review will help family physicians and endocrinologists in answering the queries of type II diabetic patients with regard to the effect of yoga practice on their medical condition.

\section{Objective}

To assess the effectiveness of practicing yoga for the management of type II diabetes mellitus in the existing literature.

\section{Inclusion Criteria}

All randomized or quasi-randomized clinical trials comparing the practice of yoga with any other type of intervention in adults with type II diabetes were included regardless of language or type of publication.
Special attention was afforded to quasi-randomized trials, as they carry a greater potential for bias.

All patients 18 years of age and older with confirmed diagnosis of diabetes mellitus type II by a physician were included. The following criteria for the diabetes diagnosis were considered for the inclusion of the studies: the World Health Organization criteria (40) or the National Diabetes Data Group standards criteria (41).

All types of known yoga practice reported in the literature were included in any comparison with another type of diabetes symptom or complication management strategies (i.e. the usual management care for diabetes or any other kind of complementary or alternative therapy). This review also included all types of comparison studies that involve comparing two or more groups with similar management but were different regarding the practice of yoga. This review also included studies which used Ayurveda as their mode of intervention. Ayurveda is an ancient Asian practice of which yoga is an integral component (42).

Glucose level control, either fasting plasma glucose (FPG) and/or glycolated hemoglobin (HbAlc), was the primary outcome measure identified in the review. Secondary outcomes identified included: change in antidiabetic medication use (as an indication for diabetes control); development of diabetic complications (including neuropathies, retinopathy, nephropathy and cardiovascular diseases); weight $(\mathrm{kg}$ ) or body mass index (BMI); lipids profile: total cholesterol, high density lipoprotein (HDL), low density lipoprotein (LDL) and triglycerides; diabetes-related mortality and any adverse effects resulted from yoga practice.

Several electronic and paper-based journal databases and indices were searched to identify studies to qualify for inclusion:

The Cochrane Metabolic and Endocrine Disorders Group: up to May 2007.

The Cochrane Central Register of Controlled

Trials (CENTRAL): up to May 2007.

MEDLINE Database (OVID): from 1966; up to the end of May 2007.

EMBASE Database (OVID): from 1980; up to the end of May 2007.

CINAHL Database (OVID): from 1982; up to the end of May 2007.

AMED Database (OVID) from 1982; up to the end of May 2007.

Up to the end of May 2007 trials registrations from (www.controlled-trials.com), Symposium, conference and colloquium proceedings and abstracts were also searched. There were no language restrictions. The research strategy was adapted mainly for Medline with minor modifications for the other databases and it was limited to human subjects only. For the complete search strategy, please see the appendix. 


\section{Hand Search}

The related studies and trials to yoga practice and diabetes were reviewed from journals that are not available electronically.

\section{Other Search Strategies}

The list of references for each of the relevant studies was searched.

Experts and authors in the field of diabetes care or yoga were contacted for any studies which were not included at this point.

\section{Methods of the Review}

This review is based on a designed protocol that can be obtained by contacting the main reviewer. The results of the searches and full texts of the included articles can also be provided by contacting the main author.

To identify suitable studies for inclusion, each trial's titles and abstracts were first reviewed. Multiple publications were grouped and evaluated as one review. The reviewing process took in to consideration the presence of adult patients with diabetes mellitus, the usage of yoga practice as the intervention in comparison to any other types of management including usual care and the presence of any of the aforementioned outcome. Full texts of relevant articles were retrieved for further assessment.

\section{Inclusion Criteria}

Adults who are 18 years of age and older with type II diabetes mellitus, diagnosed by a physician based on the criteria specified before, on a medication either oral or insulin injection, both out-patient and in-patients with stable medical condition and able to participate in the intervention.

\section{Exclusion Criteria}

Children below 18 years of age with any other type of diabetes, not diagnosed by a physician, in the critical care unit, not on any medical management for the diabetes either oral tablets or insulin injection, not mentioning a criterion for diagnosis of diabetes that was valid at the time of diagnosis and not having any medical condition that limits the physical activities from conducting the intervention.

A standardized form was developed by the reviewers to assess the quality of each trial. The data extraction form contained information on the following items: general information about the study; characteristics of the study population and participants; intervention and control characteristics and outcomes measured.
Assessing the quality of each of the trials that met the inclusion criteria was conducted independently by the two reviewers (B.A. and M.B.) and disagreement was resolved via further discussion and inter-rater agreement was calculated using the $\kappa$-statistic. The quality of each study was assessed based on quality criteria (43) that looked for the following biases:

- Selection bias: randomization and allocation concealment.

- Performance bias: any differences in care provided apart from the intervention.

- Attrition bias: any systematic differences in the withdrawals or loss to follow-up. The studies should be following the concept of intention to treat analysis (ITT) with the full explanation of the withdrawal process.

- Measurement (detection) bias: any kind of bias related to the process of reporting the outcome of the studies.

Each of the aforementioned biases was evaluated in each study and each study was graded as: (43) A (low risk of bias: all of the criteria met); $\mathrm{B}$ (moderate risk of bias: one or more criteria partly met) or $\mathrm{C}$ (high risk of bias: one or more criteria not met). Sensitivity analysis was conducted using the various levels of study quality.

\section{Data Extraction}

The extracted details regarding the relevant studies were done using a data extraction form. This process was conducted for each of the included studies. The data extraction form collected information on the following items: study general information, participant characteristics, intervention characteristics; control characteristics, outcomes characteristics and study characteristics.

\section{Data Analysis}

Statistical analysis was conducted using RevMan version 4.2.10., SAS 9.1 and comprehensive meta-analysis. Forest plots have been presented and no subgroup analysis took place. The data were planned to be summarized if they were available and of sufficient similarity and quality. The outcomes measured from each trial in our review were studied according to data-types. Summary statistics (i.e. means, standard deviations, mean difference with $95 \%$ confidence intervals) and in cases of data pooling, the weight mean difference (WMD) or standardized mean difference (SMD) were analyzed for continuous outcomes. Dichotomous outcomes were analyzed according to the odds ratios, relative risk (RR) and risk difference (RD) with 95\% confidence intervals, and numbers needed to treat (NNT). Statistical heterogeneity, using $I^{2}$ test (43) and chi-square test, were conducted when there were minimal clinical and methodological differences between the trials. 


\section{Results}

\section{Description of Studies}

\section{Studies Identified}

A total of 1815 citations were identified. Of these 1799 citations were identified through the electronic search and 16 from other sources. From the initial searches of electronic databases, 1799 citation (51 from the Cochrane Library, 846 from MEDLINE, 860 from EMBASE, 34 from CINAHL and 8 from AMED) were obtained. Total 527 studies were left after removal of the duplications and limited the studies to humans only. The titles and abstracts of these citations were reviewed. This resulted in 22 relevant citations of which the full text was obtained. After the final review of these texts, only three studies were included in this review (Figure 1) (44).

Reviewing the system for information on grey literature, the National Technical Information Service and the British National Bibliography for Report Literature revealed 16 relevant citations. Reviewing the titles and abstracts of these studies resulted in seven studies in which their full text were retrieved and gave a final relevancy of two studies.

\section{Excluded Studies}

A total of 24 studies were excluded. Nine studies were pre-post type of studies in which the participants acted as their own control but without a separated control group. Eight studies were reviews or discussion of the role of yoga practice on diabetes but they were not trials. In four of the excluded studies the intervention was not yoga practice or it was not clear that yoga practice was included and finally three of the studies were not exclusive type II diabetic patients. These were the main reasons but some of these studies were excluded for more than one reason. No other outcomes reported from any of the trails if they were not proposed originally in this review $(21,27,28,32,35,42,45-62)$ (Table 1).

\section{Studies and Participants}

A total of five studies were included in this review with 10 arms, comparing the intervention of yoga practice alone or combined with other mode of interventions. Five arms (200 participants with the control group in which two arms and 40 participants) received nothing apart from the conventional medical therapy $(63,64)$, one arm and 72 participants received recommendation on unsupervised training at home (65), one arm and 30 participants received herbal, diet and exercise (66) and the last arm with 20 participants reviewed recommendation for diet and walking exercise (20).

A total of 362 participants (200 intervention group and 164 in the control group) were analyzed. The mean age of

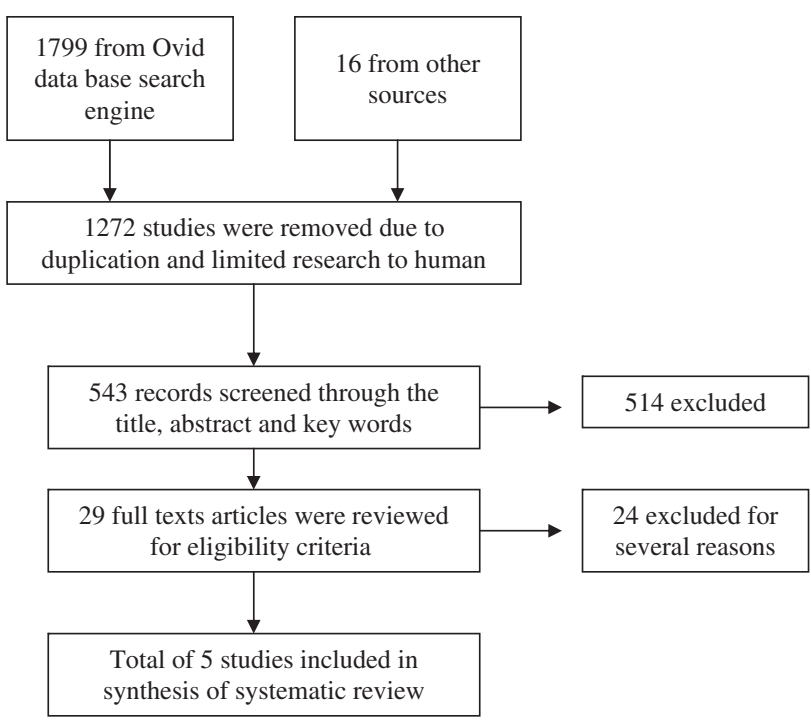

Figure 1. Flow through the different phases of systematic review.

the participants of the studies ranged from 49.9 years $( \pm 10.98)$ in the study conducted by Agrawal (65) up to 57.0 years $( \pm 7.3)$ in the control group of the Monro study (64). In one of the study conducted by Malhotra (20) they did not mention the gender ratio but for the rest of the studies there was $51 \%$ male and $49 \%$ female participants. The mean study size was 72 patients with a range from 21 to 154 patients. The dropout rate was estimated to be $12.6 \%$ of the patients that ranged from $(0 \%$ to $23 \%)$. Only one study lost $>10 \%$ (65). The mean trial duration was 14 weeks (range from 6 to 24 weeks). These studies were conducted in India and the USA.

\section{Exclusion Criteria of the Studies}

In the included studies, the following were used as exclusion criteria for participants: liver disease, pulmonary tuberculosis, malabsorption, thyrotoxicosis, alcoholism, pregnant, nursing women, psychiatric patients, nephropathy, retinopathy (proliferative), coronary artery disease, congestive cardiac failure and any serious medical condition.

\section{Interventions}

Yoga practice alone was used in three studies $(20,63,64)$ while it was combined with life style modification only in one study (65). Yoga was accompanied with lifestyle modification, diet and herbal intake in one study (66). All of the participants received training for yoga practice. While it was practiced as group in three studies, those participants in Agte and Elder $(63,66)$ studies were practicing yoga on an individual basis. The range of frequency of each session of yoga practice was different: for example two 20-min sessions per day for one of the trials (66), and three to five 90-min sessions in another (64). 
Table 1. Characteristics of the excluded studies

\begin{tabular}{|c|c|}
\hline Study & Reason for exclusion \\
\hline Malhotra et al. (21) & Trial Design: pre-post trial with no control group. \\
\hline Kaplan-Mayer (27) & $\begin{array}{l}\text { Trial Design: a review regarding yoga effects on health population: } \\
\text { general population including those with chronic diseases. }\end{array}$ \\
\hline Khalsa (28) & Trial Design: a review not a trial on yoga effect on several diseases including diabetes and other diseases. \\
\hline Singh et al. (32) & Trial Design: pre-post trial with no control group. \\
\hline Surwit and Schneider (35) & $\begin{array}{l}\text { Trial Design: pre-post trial with no control group. } \\
\text { Intervention: relaxation practice without specification of yoga. }\end{array}$ \\
\hline Doyle (45) & Trial Design: a review of yoga effect on diabetes but it was not a trail. \\
\hline Ernst (46) & Trial Design: a mini review of relaxation techniques that was not a trail. \\
\hline Head (47) & Trial Design: not a trail but a review of the effect of yoga and several other interventions on peripheral neuropathy \\
\hline Hensrud (48) & $\begin{array}{l}\text { Trial Design: a review not a trial. } \\
\text { Intervention: diet with comprehensive lifestyle therapies, involving diet, exercise, and behavioral modification. }\end{array}$ \\
\hline Bijiani et al. (51) & Trial Design: pre-post trial with no control group. \\
\hline Kathleen (52) & Trail Design: a review of the effect of alternative therapies in peripheral neuropathy. \\
\hline Kerr et al. (53) & Population: both type I and type II diabetes patients. \\
\hline Christiansen et al. (54) & $\begin{array}{l}\text { Trial Design: pre-post trial with no control group. } \\
\text { Intervention: a group of alternative medicine } \\
\text { that did not include yoga. }\end{array}$ \\
\hline Lane et al. (55) & Intervention: relaxation training that did not mention the usage of yoga. \\
\hline DiPietro et al. (56) & $\begin{array}{l}\text { Population: the participants were not diabetics. } \\
\text { Outcome: oral glucose tolerance test (OGTT). }\end{array}$ \\
\hline Mc Ginnis et al. (57) & Intervention: biofeedback-assisted relaxation with mention of the usage of yoga. \\
\hline
\end{tabular}

\section{Outcome Measures}

Primary outcome measures included in this review included FPG which was reported in four studies; and HAlc that was reported in three studies. Secondary outcomes included BMI; lipid profiles; and diabetes complications (nerve conduction) (20). None of the studies reported any adverse events.

\section{Methodological Quality of Included Studies}

The $\kappa$-score statistic was calculated to assess the agreement between the two assessors (B.A.) and (M.B.) by using SAS 9.1 software. A value of 0.6 was calculated which indicated moderate agreement (67). After discussion a final agreement was reached for all of the studies. Risk of bias was apportioned on an alphabetic score value: $\mathrm{B}$ (medium risk of bias) was given to two studies
$(64,66)$ while the remaining three studies were considered having a score of $\mathrm{C}$ (high risk of bias) $(20,63,65)$.

\section{Publication Bias}

We did not pool outcomes due to the clinical and methodological heterogeneity; however we felt it was important to estimate the magnitude of publication bias using statistical approaches. The bias coefficient from weighted regression was not significant for fasting blood glucose (Egger's test $P=0.247$ ) while the calculated failsafe $\mathrm{N}$ was 67 . HAlc (Egger's test $P=0.176$ ) was not significant and the fail-safe $\mathrm{N}$ of the might-be-missed studies to bring the effect to the null was 2. For the secondary outcomes, the bias coefficient for the cholesterol estimation was Egger's test $=0.148$, while the calculated fail-safe $\mathrm{N}$ was 47 . The coefficient for HDL was Egger's test $=0.146$ and the fail safe $\mathrm{N}$ was 85 , the 
triglyceride was insignificant as well with (Egger's test $=0.362$ ) and fail-safe $\mathrm{N}$ of 26 . We could not apply the statistical procedure for the remainder of the outcomes due to the low number of the studies included.

\section{Outcomes}

After assessing the characteristics and quality of each trial included in this review, a pooled estimate using meta-analysis was not calculated. This was mainly attributable to the high level of heterogeneity between the characteristics of studies including the specific interventions particularly the method and frequencies in conducting the intervention. Other variations existed between groups in inclusion of participants and the inconsistency in reporting outcomes between the different trials. Another important consideration is the low quality of the included studies (two of medium risk of bias and three of high risk of bias) might result in a biased overall summarization of the results (20,63-66) (Figure 2).

\section{Primary Outcomes}

\section{Fasting Plasma Glucose}

Four studies provided results on FPG. These four studies provided favorable results for the intervention in lowering FPG. The mean differences ranged from -28.8 to -41.1 in lowering the plasma glucose between the intervention and control groups, and they were all significant in three studies (63-65), however the difference in the Elder study was -3.6 and it was not significant (66).

\section{Glycolated Hemoglobin}

Three of the included studies reported the value of $\mathrm{HbAlc}$ (64-66). The mean differences indicated that the intervention lowered the level of the HbAlc. However, the results were only statistically significant in the one study (64).

\section{Secondary Outcomes}

\section{Lipid Profile}

Three of the included trials reported an effect on lowering cholesterol level (63-66). The mean differences for the effect ranged from -9.1 to -35.9 which were significant in all of the trials except one (66). These trials reported outcomes additionally for HDL suggesting that yoga had a positive effect in increasing the level of HDL. The mean difference magnitude was 8.49 and 10.0 and they were both statistically significant $(63,65)$. The Elder trial reported opposite effect in increasing HDL by a mean difference magnitude of -1.30 and a $95 \%$ CI of $(-5.32,2.72)(66)$. However, these trials were consistent in their reporting of the beneficial effect yoga has on triglyceride levels in which all of them reported negative mean differences ranging from -8.00 to -31.01 . Only two of the trials reported the effect of yoga on LDL $(65,66)$, one showed a lowering effect while the other evidenced a harmful effect by increasing the LDL, however this effect was not significant.

\section{Weight and Body Mass Index}

The study conducted by Agrawal reported a minimal drop in the mean difference of the weight of -0.17 which was not statistically significant (65). The Elder study found a two-BMI unit (66).

\section{Diabetes-Related Complication}

The only effect of yoga practice on diabetes complications identified was improvement in the nerve conduction velocity by Malhotra (20). They reported an improvement in nerve conduction velocity in both arms in the yoga group, but the change was not statistically significant.

\section{Discussion}

This review addressed the effect of yoga practice on patients with type II diabetes. Despite an extensive review of the literature via different methods, we could only include five trials that compared those practicing yoga alone or with other co-interventions to a control group. To find high-quality trials (RCTs), we excluded a large number of pre-post trials, and unfortunately the included studies were not of high quality.

Yoga practice varied in type and duration between trials; while it was only for 40 days in Malhotra study, it increased to six months in the Elder trial $(20,66)$. Among those studies who reported the details of the yoga practice, the mean time of yoga practice was $300 \mathrm{~min}$ per week (approximately $45 \mathrm{~min}$ per day).

The trials we reviewed suggested a general beneficial effect of yoga on diabetic patients. These effects were most prominent in FPG levels and lipid profiles. The results were significant in the majority of the trials. Certain long-term parameter, such as $\mathrm{HbAlc}$ was reported to improve slightly with the practice of yoga. However this did not increase statistically significant levels in two of the three trials in which it was reported. Other outcomes showed a favorable but not significant effect in reducing BMI units and body weight. Only one study tested the effect of yoga on diabetic-related complications (nerve conduction velocity) that showed no significant improvement from the control group.

The results suggest favorable effects of yoga on the short-term parameters related to diabetes but not necessarily for the long-term outcomes. This might be attributable to the short duration of the trials which were not able to detect a long-term effect and to the low power 


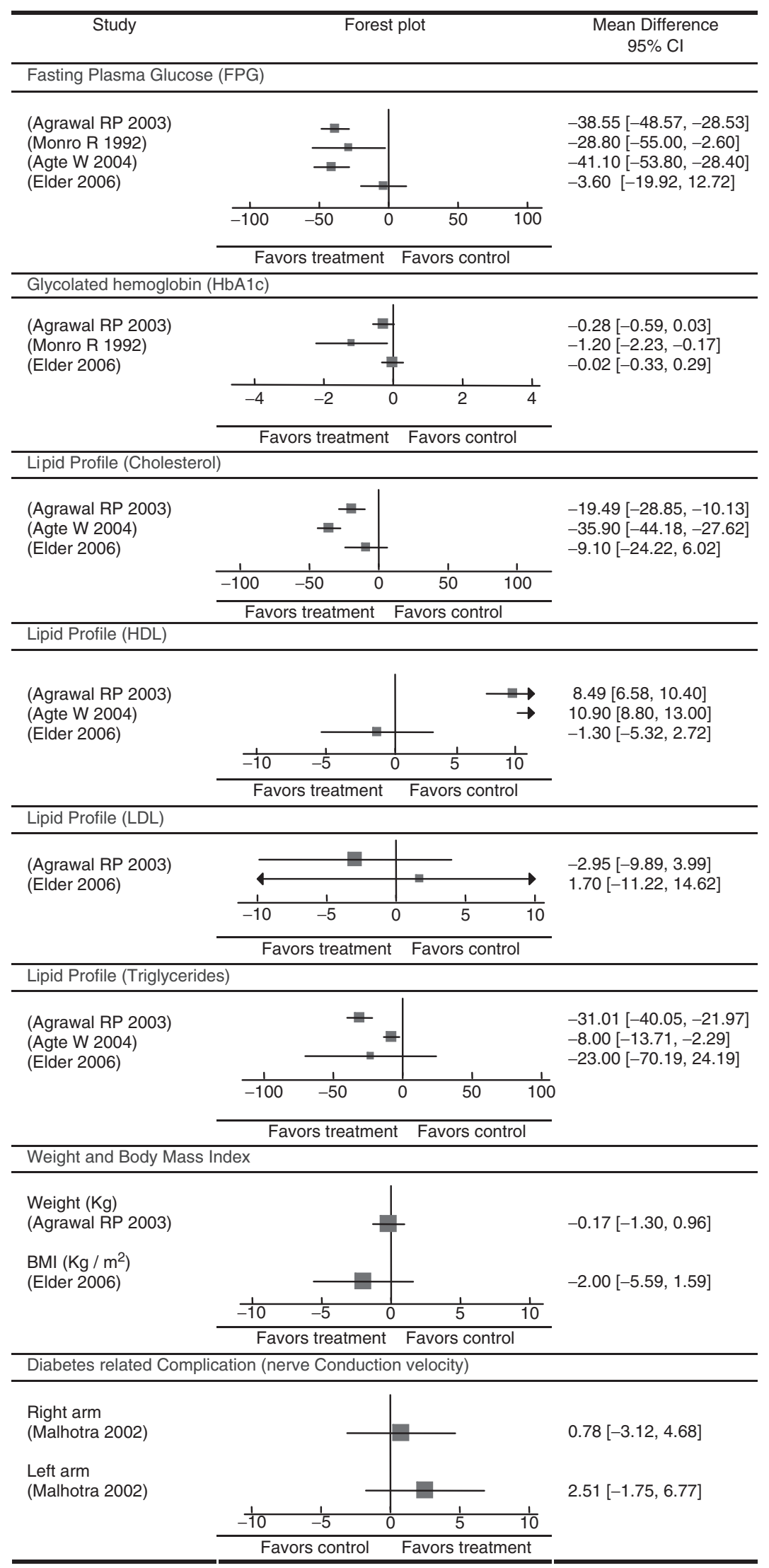

Figure 2. Forest plots of the primary and secondary outcomes of the included studies. 
of each trial due to a small number of participants. Also, these long-term parameters require consistency in practicing yoga which raises the issue of non-adherence of the patients practicing yoga as they were scheduled to do so.

The rest of the proposed outcomes were not able to be retrieved, mainly those related to long-term complication and mortality. The reasons could be the result of the eligibility criteria of the trials since they excluded any patient with poor health status or having any kind of complication and the short period of the trials.

The results of the sensitivity analysis showed mixed results: for the primary outcomes there were no changes in the effect produced by yoga, and for the secondary outcomes the effect was null with the exclusion of high risk trials.

In this review, pooling the results from the different studies would not be of a scientific value due to the clinical heterogeneity between the studies and the risk of bias due to low quality of the studies. The low quality of included studies could be resulted from methodological deficiencies, from inappropriate reporting, or both. Methodological deficiencies such as allocation concealment, randomization, selection bias, statistical analysis, usage of multiple interventions and adjustment for confounders were noticed with varying degrees among included studies.

Publication bias assessed for the different outcomes reported by the trials to assess if there were any evidence suggesting a bias that would lead to publication of specific trials and not the other. The bias coefficient from weighted regression was not significant for all of the included studies and the number of studies of negative results that would bring the effect of yoga practice on the primary outcome, FPG and HBA1c to the null were 67 and 2. While it is unlikely that we were not able to include any of the 67 studies for the FPG, not including any of the two studies to bring the effect of yoga on HbAlc to the null is possible.

In summary, yoga showed favorable outcomes among patients with diabetes type II. These improvements were mainly among short term or immediate diabetes outcomes and not all of the improvements were statistically significant. Factors like the study quality and intervention characteristics should be taken into consideration.

\section{Conclusions}

None of the included trials report any side effects for yoga practice. Short-term benefits for patients with diabetes might be achieved from practicing yoga. Further analysis of practice details and frequency are needed. The trials in our review had methodological quality considerations, and the trials' reported benefits have not been confirmed by large trials of high-quality. A definitive recommendation for physicians to encourage their patients to practice yoga cannot be reached at the present.

The important recommendation that can be drawn from this systematic review is the need for well-designed large randomized clinical trials to assess the effectiveness of yoga on diabetes type II. These trials should concentrate on the methodological quality and the specification of the characteristics of yoga practice. These trials should avoid including different interventions in the same arm which may cause confounding effects on the relationship between yoga and diabetes. If researchers want to include other types of co-interventions in the trial they should take into consideration methodological issues such as sample size and study power and to include subgroup analysis. Studying the long-term impact of yoga and its side effects is needed and likely stipulates standardized monitoring and reporting. Trials are recommended to follow CONSORT statement in reporting.

\section{Acknowledgements}

The authors would like to thank professors David Moher and Dean Fergusson for their unlimited support.

\section{References}

1. Emslie M, Campbell M, Walker K. Family Medicine Complementary therapies in a local healthcare setting. Part 1: is there real public demand? Complement Ther Med 1996;4:39-42.

2. MacLennan AH, Wilson DH, Taylor AW. The escalating cost and prevalence of alternative medicine. Prevent Med 2002;35:166-73.

3. Lew-Ting CY. Who uses non-biomedical, complement and alternative health care? Sociodemographic undifferentiation and the effects of health needs. Taiwan J Public Health 2003;22:155-66.

4. Lim MK, Sadarangani P, Chan HL, Heng JY. Complementary and alternative medicine use in multiracial Singapore. Complement Ther Med 2005;13:16-24.

5. Tindle HA, Davis RB, Phillips RS, Eisenberg DM. Trends in use of complementary and alternative medicine by US adults: 1997-2002. Alternat Ther Health Med 2005;11:42-9.

6. Dunning T. Complementary therapies and diabetes. Complement Ther Nurs Midwifery 2003;9:74-80.

7. Yeh GY, Eisenberg DM, Davis RB, Phillips RS. Use of complementary and alternative medicine among persons with diabetes mellitus: results of a national survey. Am J Public Health 2002;92:1648-52.

8. Bell RA, Suerken CK, Grzywacz JG, Lang W, Quandt S.A, Arcury T.A. Complementary and alternative medicine use among adults with diabetes in the United States. Alternat Ther Health Med 2006;12:16-22.

9. Shapiro D, Cook IA, Davydov DM, Ottaviani C, Leuchter AF, Abrams M. Yoga as a complementary treatment of depression: effects of traits and moods on treatment outcome. Evid Based Complement Alternat Med 2007;4:493-502.

10. Lloyd P, Lupton D, Wiesner D, Hasleton S. Choosing alternative therapy: an Australian study of sociodemographic characteristics and motives of patients resident in Sydney. Australas $J$ Public Health 1993;72:135-44.

11. MacLennan A, Wilson P, Taylor A. Prevalence and cost of alternative medicines in Australia. Lancet 1996;347:569-73.

12. Hunter A. Why do people see natural therapists? A review of the surveys. Diversity 1997;10:15-19.

13. Ramaratnam S, Sridharan K. Yoga for epilepsy. In: The Cochrane Library, Issue 1. Oxford: update software, 2000. 
14. Sabina AB, Williams A-, Wall HK, Bansal S, Chupp G, Katz DL. Yoga intervention for adults with mild-to-moderate asthma: a pilot study. Ann Allergy, Asthma Immunol 2005;94:543-8.

15. Gupta N, Khera S, Vempati RP, Sharma R, Bijlani RL. Effect of yoga based lifestyle intervention on state and trait anxiety. Indian J Physiol Pharmacol 2006;50:41-7.

16. Guanacino JL, Savino S, Edelstein S. Yoga participation is beneficial to obesity prevention, hypertension control, and positive quality of life. Top Clin Nutr 2006;21:108-13.

17. Khattab K, Khattab AA, Ortak J, Richardt G, Bonnemeier H. Iyengar Yoga increases cardiac parasympathetic nervous modulation among healthy yoga practitioners. Evid Based Complement Alternat Med 2007;4:511-17.

18. Van Puymbroeck M, Payne LL, Hsieh P-. A phase I feasibility study of yoga on the physical health. Evid Based Complement Alternat Med 2007;4:519-29.

19. Yang K. A review of yoga programs for four leading risk factors of chronic diseases. Evid Based Complement Alternat Med 2007:4:487-91.

20. Malhotra V, Singh S, Tandon OP, Madhu SV, Prasad A, Sharma SB. Effect of Yoga asanas on nerve conduction in type 2 diabetes. Indian J Physiol Pharmacol 2002;46:298-306.

21. Malhotra V, Singh S, Tandon OP, Sharma SB. The beneficial effect of yoga in diabetes. Nepal Med Coll J 2005;7:145-7.

22. Manyam BV, Sahay BK, Sahay RK. Lifestyle modification in management of diabetes mellitus. J Indian Med Assoc 2002;100: $178-80$.

23. Manyam By. Diabetes mellitus, Ayurveda, and yoga. Comment. J Altern Complement Med 2004;10:223-25.

24. Sahay BK. Role of yoga in diabetes. J Assoc Physicians India 2007;55:121-6.

25. Dham S, Shah V, Hirsch S, Banerji MA. The role of complementary and alternative medicine in diabetes. Curr Diab Rep 2006:6:251-58.

26. Gupta SM. Modern medicine and yoga. J Intern Med India 2001;4:155-6.

27. Kaplan-Mayer G. Get moving with yoga. Diabetes Self Manag 2003;20:28, 31-3.

28. Khalsa SBS. Yoga as a therapeutic intervention: a bibliometric analysis of published research studies. Indian J Physiol Pharmacol 2004:48:269-85.

29. Nagarathna R, Nagendra HR. Integrated approach of yoga therapy in the management of diabetes mellitus. Proceedings of the Ninth Annual Conference of the IEEE Engineering in Medicine and Biology Conference. New York, NY, USA: IEEE 1987:1593-94.

30. Nayak NN, Shankar K. Yoga: a therapeutic approach. Phys Med Rehabil Clin N Am 2004;15:783-98.

31. Shembekar AG, Kate SK. Yoga exercises in the management of diabetes mellitus. J Diabetic Assoc India 1980;20:167-71.

32. Singh S, Malhotra V, Singh KP, Madhu SV, Tandon OP. Role of Yoga in modifying certain cardiovascular functions in type 2 diabetic patients. J Assoc Physicians India 2004;52:203-06.

33. Stevens DL. The use of complementary and alternative therapies in diabetes. Clin Fam Prac 2002;4:911-28.

34. Innes KE, Bourguignon C, Taylor AG. Risk indices associated with the insulin resistance syndrome, cardiovascular disease, and possible protection with yoga: a systematic review. J Am Board Fam Pract 2005; 18:491-519.

35. Surwit RS, Schneider MS. Role of stress in the etiology and treatment of diabetes mellitus. Psychosom Med 1993;55:380-93.

36. Jablon SL, Naliboff BD, Gilmore SL, Rosenthal MJ. Effects of relaxation training on glucose tolerance and diabetic control in type II diabetes. Appl Psychophysiol Biofeed 1997;22:155-69.

37. Da Silva GD, Lorenzi-Filho G, Lage LV. Effects of yoga and the addition of Tui $\mathrm{Na}$ in patients with fibromyalgia. $J$ Alternat Complement Med 2007;13:1107-113.

38. American Diabetes Association. Unproven therapies (Position Statement). Diabetes Care 2004;27:S135.

39. World Health Organisation. Diabetes: the cost of diabetes. www.who.int/mediacentre/factsheets/fs23 6/en! (accessed February $15,2007)$

40. World Health Organization. World Health Report 1999 (WHO 2002).
41. NDDG. National Diabetes Data Group. Classification and diagnosis of diabetes mellitus and other categories of glucose intolerance. Diabetes 1979;28:1039-57.

42. Mamtani R, Mamtani R. Ayurveda and yoga in cardiovascular diseases. Cardiol Rev 2005;13:155-62.

43. Higgins JPT, Green S (eds). Cochrane Handbook for Systematic Reviews of Interventions. 4.2.6 [updated September 2006] edition. In: The Cochrane Library, Issue 4. Chichester, UK: John Wiley \& Sons, Ltd., 2006.

44. Moher D, Cook DJ, Eastwood S, Olkin I, Rennie D, Stroup D, for the QUOROM group. Improving the quality of reporting of metaanalysis of randomized controlled trials: the QUOROM statement. Lancet 1999;354:1896-900.

45. Doyle C. Can yoga take the strain. Daily Telegraph 1988;12:26-9.

46. Ernst E. Mini-review: relaxation techniques for diabetic patients a re-appraisal of an old form of treatment. Eur J Phys Med Rehabil 1995;5:54-55.

47. Head KA. Peripheral neuropathy: pathogenic mechanisms and alternative therapies. Alternat Med Review 2006;11:294-329.

48. Hensrud DD. Dietary treatment and long-term weight loss and maintenance in type 2 diabetes. Obes Res 2001;9 (Suppl 4): $348 \mathrm{~S}-53 \mathrm{~S}$.

49. Irace L, larussi D, Guadagno I, De Rimini ML, Lucca $P$, Spadaro $\mathrm{P}$, et al. Left ventricular function and exercise tolerance in patients with type II diabetes mellitus. Clin Cardiol 1998; 21:567-571.

50. Jam SC, Uppal A, Bhatnagar SO, Talukdar B. A study of response pattern of non-insulin dependent diabetics to yoga therapy. Diabetes Res Clin Pract 1993;19:69-74.

51. Bijlani RL, Vempati RP, Yadav RK, Ray RB, Gupta V, Sharma R, et al. A brief but comprehensive lifestyle education program based on yoga reduces risk factors for cardiovascular disease and diabetes mellitus. J Altern Complement Med 2005;1 1:267-74.

52. Kathleen A. Head. Peripheral neuropathy: pathogenic mechanisms and alternative therapies. Alternat Med Review 2006;11:294-329.

53. Kerr D, Gillam E, Ryder J, Trowbridge S, Cavan D, Thomas P. An Eastern art form for a Western disease: randomised controlled trial of yoga in patients with poorly controlled insulin-treated diabetes. Practic Diabet Int 2002;19:164-66.

54. Christiansen MP, Linfoot PA, Neese RA, Hellerstein MK. Effect of dietary energy restriction on glucose production and substrate utilization in type 2 diabetes. Diabetes 2000;49:1691-99.

55. Lane JD, McCaskill CC, Ross SL, Feinglos MN, Surwit RS Relaxation training for NIDDM. Predicting who may benefit. Diabetes Care 1993;16:1087-94.

56. DiPietro L, Seeman TE, Stachenfeld NS, Katz LD, Nadel ER. Moderate-intensity aerobic training improves glucose tolerance in aging independent of abdominal adiposity. $J$ Am Geriatr Soc 1998:46:875-79.

57. McGinnis RA, McGrady A, Cox SA, Grower-Dowling KA. Biofeedback-assisted relaxation in type 2 diabetes. Diabetes Care 2005;28:2145-9.

58. Naruka JS, Mathur R, Mathur A. Effect of pranayama practices on fasting blood glucose and serum cholesterol. Indian $\mathrm{J} \mathrm{Med} \mathrm{Sci}$ 1986:40:149-52.

59. Singh S, Malhotra V, Singh KP, Sharma SB, Madhu SV, Tandon OP. A preliminary report on the role of yoga asanas on the oxidative stress in non-insulin dependent diabetes mellitus. Indian J Clin Biochem 2001;16:216-20.

60. Diaz-Nieto L, Galan-Cuevas S, Femandez-Pardo G. A self-care group in diabetes mellitus type 2. Salud Publica Mex 1993;35:169-76.

61. Tsujiuchi T, Kumano H, Yoshiuchi K, He D, Tsujiuchi Y, Kuboki $\mathrm{T}$, et al. The effect of Qi-gong relaxation exercise on the control of type 2 diabetes mellitus: a randomized controlled trial. Diabetes Care 2002;25:241-2.

62. Vanelli M, Chiari G, Gugliotta M, Capuano C, Giacalone T, Gruppi L, et al. Diabetes and alternative medicine: diabetic patients experiences with Ayur-Ved, 'clinical ecology' and 'cellular nutrition' methods. Minerva Pediatr 2002;54:165-9.

63. Agte VV, Tarwadi K. Sudarshan kriya yoga for treating type 2 diabetes: a preliminary study. Alternat Complement Ther 2004:10:220-2. 
64. Monro R, Power J, Coumar A, Nagarathna R, Dandona P. Yoga therapy for NIDDM: a controlled trial. Complement Med Res 1992;6:66-8

65. Agarwal RP, Aradhana R, Hussain S, Beniwal R, Sabir M, Kochar $\mathrm{DK}$, et al. Influence of yogic treatment on quality of life outcomes, glycaemic control and risk factors in diabetes mellitus. International Journal of Diabetes in Developing Countries 2003;23:130-4.
66. Elder C, Aickin M, Bauer V, Cairns J, Vuckovic N. Randomized trial of a whole-system ayurvedic protocol for type 2 diabetes. Altern Ther Health Med 2006;12:24-30.

67. Landis RJ, Koch GG. The measurement of observer agreement for categorical data. Biometrics 1977;33:159-74.

Received December 4, 2007; accepted April 4, 2008 


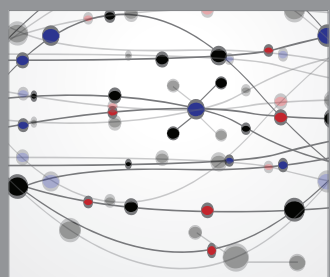

The Scientific World Journal
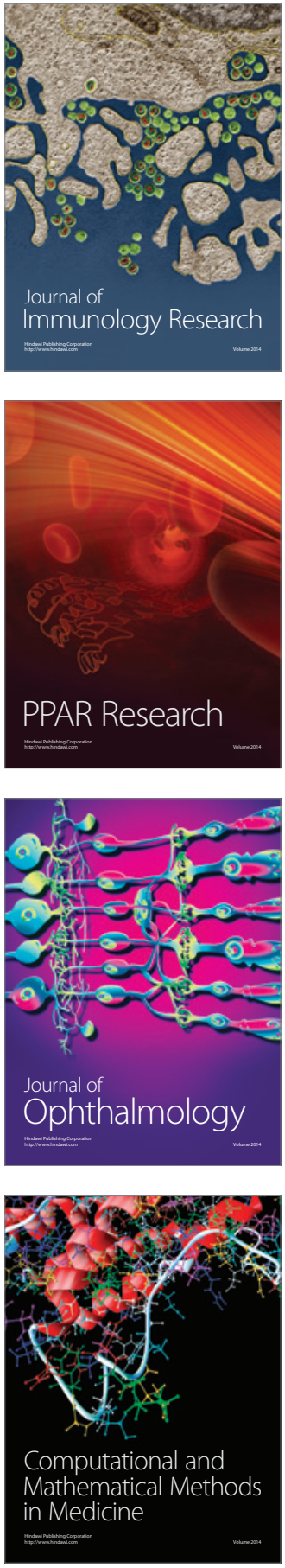

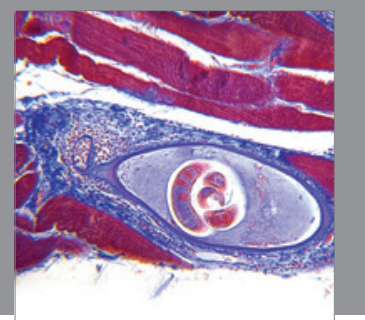

Gastroenterology

Research and Practice
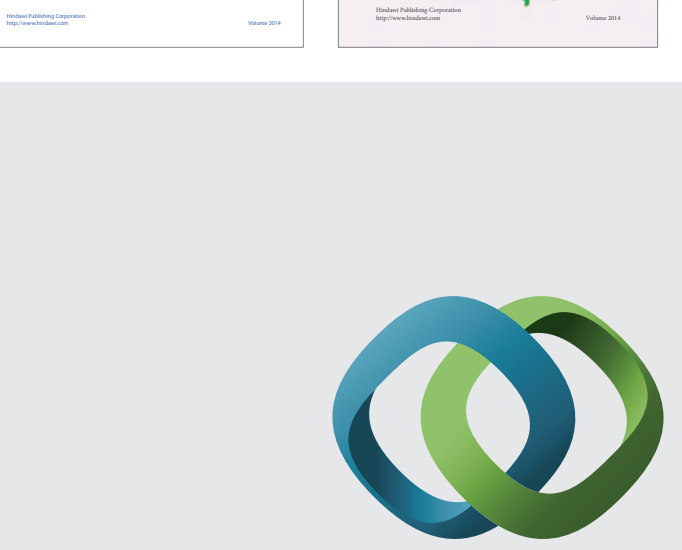

\section{Hindawi}

Submit your manuscripts at

http://www.hindawi.com
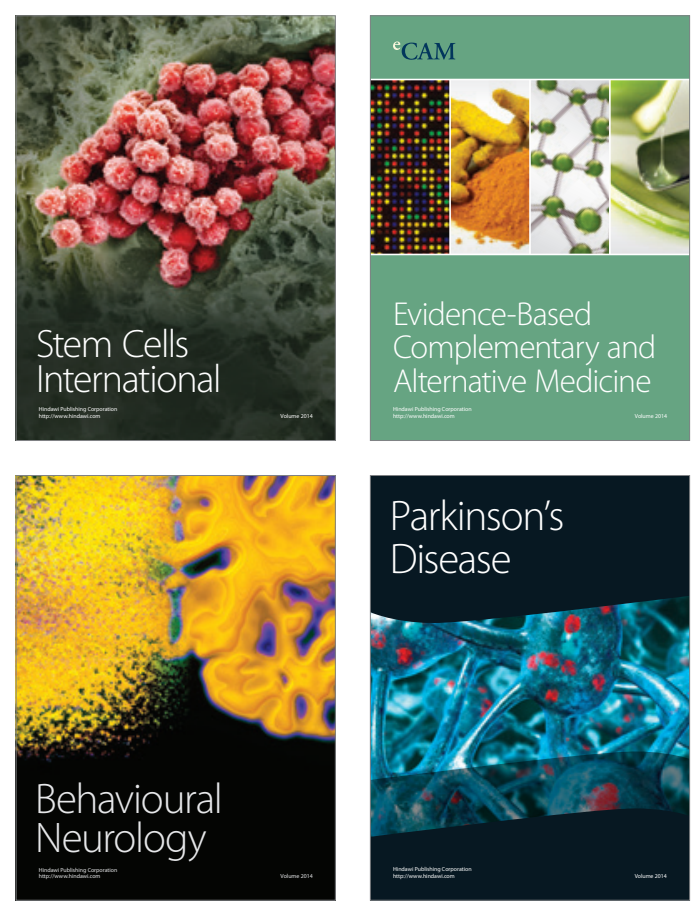

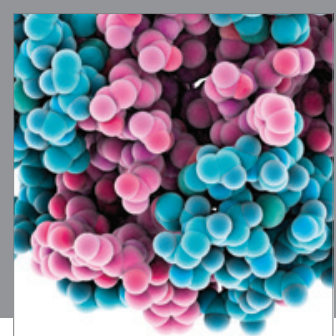

Journal of
Diabetes Research

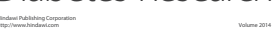

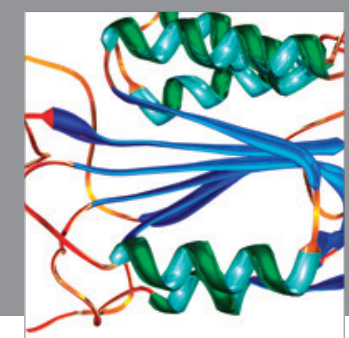

Disease Markers
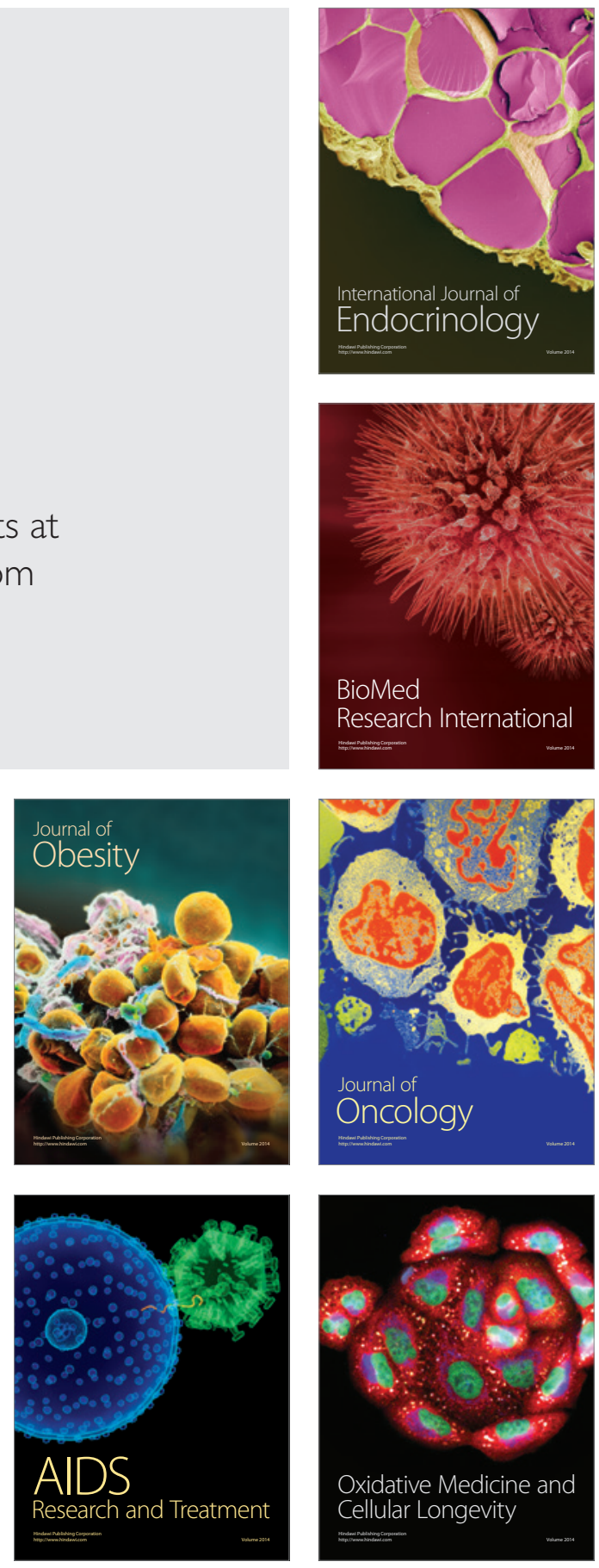